\title{
Early Appropriate Parenteral Antimicrobial Treatment of Complicated Skin and Soft Tissue Infections Caused by Methicillin-Resistant Staphylococcus aureus
}

\author{
Lena M. Napolitano
}

\begin{abstract}
Background: Complicated skin and soft tissue infections (cSSTIs) are a major clinical problem, in part because of the increasing resistance of infecting bacteria to our current antibiotic therapies. Prompt appropriate treatment of infections in hospitalized patients reduces the mortality rate. Furthermore, appropriate and timely antibiotic therapy improves outcomes for cSSTIs caused by methicillin-resistant Staphylococcus aureus (MRSA). This review delineates factors to consider in the choice of initial antibiotic treatment for cSSTIs and describes the antimicrobial agents available or under clinical development for the treatment of cSSTIs caused by MRSA. Methods: Review of the pertinent literature and recommendations.

Results: The choice of antimicrobial agent for empiric treatment of cSSTIs should be guided by the site and type of infection, the presence of an immunocompromised state or neutropenia, and risk factors for hospitalacquired MRSA (HA-MRSA) or community-associated MRSA (CA-MRSA) infection. Most CA-MRSA strains remain susceptible to ciprofloxacin, clindamycin, gentamicin, and trimethoprim/sulfamethoxazole, although resistance to clindamycin can emerge during treatment. Of the agents available for the treatment of HA-MRSA cSSTIs, vancomycin has been the reference standard, but clinical failures have been reported increasingly. Alternative agents for HA-MRSA include linezolid, which has been well-studied for treatment of cSSTIs, as well as daptomycin and tigecycline. A number of antibiotic agents are undergoing clinical trials or are under development for the treatment of cSSTIs caused by MRSA.

Conclusions: Severe and progressive cSSTIs should be treated promptly with appropriate antibiotic agents. The choice of agent should be guided by a number of factors, including suspected CA-MRSA or HA-MRSA infection. Available agents should be evaluated carefully for efficacy in the treatment of MRSA cSSTIs.
\end{abstract}

$\mathbf{T}$ HE INITIAL MANAGEMENT of complicated skin and soft tissue infections (cSSTIs) should include the collection of specimens for culture and antimicrobial susceptibility testing from all abscesses or purulent lesions [1]. Culture and susceptibility findings are useful both for individual patient management and for monitoring of local patterns of antimicrobial resistance [1]. Physicians and other healthcare workers cannot predict accurately if an SSTI is attributable to methicillin-resistant Staphylococcus aureus (MRSA) [2]. A prospective observational study of 176 emergency department patients presenting with purulent wounds and abscesses documented that physician suspicion of MRSA had a sensitivity of only $80 \%$ (95\% confidence interval [CI], 71, 87) and a specificity of $23.6 \%(95 \%$ CI 14,37$)$ for the presence of MRSA on wound culture, with a positive likelihood ratio (LR) of $1.0(95 \%$ CI $0.9,1.3)$ and a negative LR of 0.8 (95\% CI 0.5, 1.3). The prevalence of such infections was $64 \%$ [2].

The research reviewed in this paper demonstrates that timely and appropriate empiric antibiotic therapy improves the outcomes of patients with serious infections, including SSTIs caused by MRSA [3,4]. Empiric antibiotic therapy should be initiated in all patients with cSSTIs. Intravenous (IV) broad-spectrum antimicrobial therapy should be given when an infection is severe or progresses rapidly, when there

Department of Surgery, University of Michigan Health System, Ann Arbor, Michigan.

Lena M. Napolitano, M.D., FACS, FCCP, FCCM, received an honorarium from Pfizer Inc. in connection with the development of this manuscript. Editorial support was provided by AlphaMedica, Inc., and was funded by Pfizer Inc. 
are signs of systemic illness, when the patient has co-morbidities or is immunosuppressed, when the patient is very old or young, when an abscess cannot be drained completely, or when the infection does not respond to incision and drainage [1]. A number of antimicrobial agents are now available for the treatment of cSSTIs caused by MRSA. This paper discusses considerations in the choice of initial therapy and the agents available and under development for the treatment of these infections.

\section{Benefits of Prompt Appropriate Antibiotic Therapy for Serious Infections}

\section{Appropriate initial treatment of infections in the intensive care unit reduces deaths}

Inadequate treatment of infections in patients in the intensive care unit (ICU) contributes to in-hospital death. A 1999 surveillance study of 2,000 consecutive eligible patients with infections necessitating ICU admission found that the hospital mortality rate in those receiving inadequate antimicrobial treatment was significantly greater than that of patients without this risk factor $(52.1 \%$ vs. $12.2 \%$; relative risk [RR] 4.26; 95\% CI 3.52, 5.15; p < 0.001) (Fig. 1) [3]. In this study, oxacillin-resistant $S$. aureus was the most common gram-positive bacterial pathogen isolated from individuals receiving inadequate initial therapy [3]. Similar findings were reported in patients with ventilator-associated pneumonia (VAP) [5] or sepsis [6]. A study of ICU patients found that the higher mortality rate associated with inappropriate initial therapy is still observed when antibiotics are switched from an inappropriate to an appropriate agent [7].

\section{Delayed appropriate treatment associated with longer hospital stay}

In addition to lowering the mortality rate, timely appropriate treatment may reduce hospital length of stay (LOS).

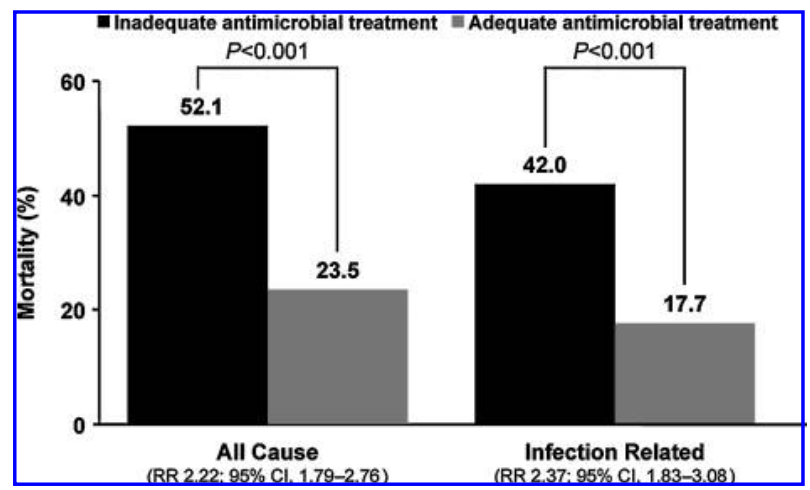

FIG. 1. In 2,000 hospitalized patients with infections requiring intensive care unit (ICU) admission, hospital mortality rate of patients receiving inadequate antimicrobial treatment was significantly greater than that of patients without this risk factor $(52.1 \%$ vs. $12.2 \%$; relative risk [RR] 4.26; 95\% confidence interval [CI] 3.52, 5.15; $\mathrm{p}<0.001)$. The infection-related mortality rate also was statistically greater among infected patients receiving inadequate antimicrobial treatment $(42.0 \%)$ than in infected patients receiving adequate antimicrobial treatment (17.7\%) (RR 2.37; 95\% CI 1.83, 3.08; $\mathrm{p}<0.001)$ [3].
A study of 167 patients with S. aureus bacteremia found that $33.3 \%$ of patients with delayed appropriate treatment died vs. $23 \%$ of patients who received early appropriate treatment $(\mathrm{p}=0.05)$ [8]. In multivariable analyses, delayed treatment was an independent predictor of infection-related death (odds ratio [OR] 3.8; 95\% CI 1.3, 11.0; $\mathrm{p}=0.01$ ), and the adjusted mean LOS was longer in the delayed treatment than the early treatment group (20.2 days vs. 14.3 days; $\mathrm{p}=0.05$ ) [8]. By logistic regression analysis, MRSA infection was the most significant predictor of delayed appropriate treatment (OR 8.3; 95\% CI 2.6, 16.8); 42 of 48 episodes of delayed treatment involved MRSA infection. Appropriate treatment was delayed in these episodes because patients were treated initially with an antibiotic that lacked activity against the MRSA strain [8].

\section{Inappropriate treatment of CA-MRSA cSSTI associated with treatment failure}

In a retrospective study of 492 patients with communityassociated MRSA (CA-MRSA) SSTIs, 95\% of the episodes treated with an active antibiotic within $48 \mathrm{~h}$ were treated successfully, compared with an $87 \%$ rate of successful treatment in patients who did not receive an active antibiotic $(p=0.001)$ [4]. By logistic regression analysis, failure to initiate active antimicrobial therapy within $48 \mathrm{~h}$ of presentation was the only independent predictor of treatment failure (adjusted OR 2.80; 95\% CI 1.26, 6.22; $\mathrm{p}=0.011$ ) [4]. Similarly, in a study of patients admitted to the hospital with MRSA sterile-site infection, multivariable analysis found inappropriate antimicrobial treatment to be an independent risk factor for in-hospital death (adjusted OR 1.92; 95\% CI 1.48, 2.50; $\mathrm{p}=0.013$ ) [9].

An empiric treatment algorithm for SSTI in the setting of escalating CA-MRSA was examined in emergency room patients. The algorithm promoted the use of antibiotics that are likely active against CA-MRSA along with early incision and drainage of abscesses. Clinical failure occurred in only $3 \%$ of the patients treated according to the algorithm, compared with $62 \%$ of those not so treated $(\mathrm{p}<0.001)$. Furthermore, among patients who underwent immediate incision and drainage, initial treatment with antibiotics active in vitro against the MRSA isolate was associated with a lower clinical failure rate than treatment with inactive antibiotics (0 vs. $67 \%$; $\mathrm{p}<0.001$ ) [10]. However, in one recent study of $117 \mathrm{pa}-$ tients with CA-MRSA and methicillin-sensitive $S$. aureus (MSSA) skin infections, initial receipt of an antibiotic inactive against the infecting strain did not predict non-response to treatment at day $30[11]$.

\section{Considerations in Choice of Initial Therapy}

\section{Site and type of infection}

The choice of empiric antimicrobial therapy for cSSTIs is guided by a number of factors. In the case of surgical site infection (SSI), the type and site of the surgical procedure dictate which pathogens are suspected. Infections following surgery of the gastrointestinal or genitourinary tract may be either monomicrobial or mixed, and may be caused by grampositive or gram-negative bacteria. By contrast, infections following clean operations in other parts of the body typically are caused by gram-positive pathogens [12]. 
Certain community presentations of skin infection increasingly are caused by MRSA, including impetigo and necrotizing fasciitis [13]. Diabetic foot infections typically involve $S$. aureus; other organisms may be present, but possibly as colonizers, not pathogens [12].

\section{Immunocompromised and neutropenic patients}

Immunocompromised patients are, of course, at higher risk of infection and less able to control local infection [13]. Immunocompromised or neutropenic patients should be treated with empiric, broad-spectrum antibiotics at the first clinical signs of infection, including fever [13]. Invasive skin infections with CA-MRSA have been reported in solid organ transplant recipients. These reports emphasize the clinical importance of considering CA-MRSA as a causative pathogen in the differential diagnosis of cSSTIs in organ transplant recipients [14].

In selecting empiric antibiotic therapy for skin infection in neutropenic patients, consideration should be given to adequate coverage against virulent and resistant gram-positive organisms, including MRSA, vancomycin-resistant enterococci (VRE), or penicillin-resistant Streptococcus pneumoniae. Linezolid or daptomycin may be acceptable alternatives to vancomycin [13].

\section{Risk for HA-MRSA or CA-MRSA infection}

All patients should be assessed for increased risk of hospital-acquired MRSA (HA-MRSA) and CA-MRSA skin infection. Risk factors for HA-MRSA infection include isolation of MRSA two or more days after hospitalization; a history of hospitalization, surgery, dialysis, or residence in a long-term care facility within the previous year; the presence of a permanent indwelling catheter or percutaneous medical device; or previous isolation of MRSA [15].

Risk factors for CA-MRSA are less clear than those for HAMRSA. Outbreaks of skin infections caused by CA-MRSA strains have been reported in community residents who lack the typical risk factors for MRSA infection. These patients include prison inmates, injection drug users, Native American populations, men who have sex with men, and children [13]. A list of persons at risk for SSTIs caused by CA-MRSA is

Table 1. Persons at Risk for Skin and Soft Tissue InFeCtions CAUSED by COMMUnity-Associated Methicillin-Resistant StaphyLOcoccus aureus

$\begin{array}{cl}\text { Household contacts of } & \text { Intravenous drug users } \\ \text { patients with proven } & \text { Incarcerated persons } \\ \text { CA-MRSA infection } & \text { Athletes, particularly those } \\ \text { Children } & \text { involved in contact sports } \\ \text { Day care center contacts of } & \text { Native Americans } \\ \text { hospitalized patients } & \text { Pacific Islanders } \\ \text { with MRSA infections } & \text { Persons with previous } \\ \text { Men who have sex } & \text { CA-MRSA infection } \\ \text { with men } & \\ \text { Military personnel } & \end{array}$

Reproduced with permission from reference 16. Copyright (@) 2007 Massachusetts Medical Society. All rights reserved.

CA-MRSA $=$ community-associated methicillin-resistant Staphylococcus aureus; MRSA = methicillin-resistant S. aureus.
Table 2. Recommended Antimicrobial Therapy fOR Skin and Soft Tissue Infections in Adults Caused by Methicillin-Resistant Staphylococcus auReus ${ }^{\mathrm{a}}$

\begin{tabular}{ll}
\hline Intravenous antibiotics & $30 \mathrm{mg} / \mathrm{kg} / \mathrm{d}$ in two doses \\
Vancomycin & $600 \mathrm{mg} \mathrm{q} 12 \mathrm{~h}$ \\
Linezolid & $600 \mathrm{mg} \mathrm{q} 8 \mathrm{~h}$ \\
Clindamycin & $4 \mathrm{mg} / \mathrm{kg} \mathrm{q} 24 \mathrm{~h}$ \\
Daptomycin & \\
Oral antibiotics & $600 \mathrm{mg} \mathrm{bid}$ \\
Linezolid & $300-450 \mathrm{mg}$ tid \\
Clindamycin & $100 \mathrm{mg}$ bid \\
Doxycycline or minocycline & 1 or 2 double-strength \\
Trimethoprim/ & tablets bid \\
$\quad$ sulfamethoxazole & \\
\hline
\end{tabular}

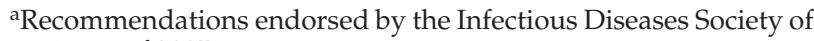
America as of 2005.

Adapted from references 12 and 13.

given in Table 1 [16]. Outbreaks of furunculosis caused by CA-MSSA and by CA-MRSA may occur in families and in other settings involving close personal contact, such as sports teams. Inadequate personal hygiene and contact with infected individuals are predisposing factors [13].

\section{Initial broad-spectrum therapy and de-escalation}

All patients who present with cSSTIs should receive broad-spectrum antimicrobial therapy, including mandatory coverage for MRSA. Patients who present to the hospital with severe infection or infection progressing despite antibiotic therapy should be treated aggressively. In these cases, if $S$. aureus is cultured, the clinician should assume the organism is resistant and administer an agent effective against MRSA, such as vancomycin, linezolid, or daptomycin. Stepdown to other agents for MRSA infection, such as minocycline or trimethoprim/sulfamethoxazole (TMP/SMX), may be considered according to the susceptibility findings and initial clinical response [13].

\section{Choice of Treatment for MRSA cSSTI}

The choice of treatment agent for MRSA cSSTI depends on the type of infection and suspected pathogens [12]. Table 2 presents recommendations endorsed by the Infectious Diseases Society of America (2005) for MRSA SSTI treatment in adults [13].

\section{HA-MRSA and CA-MRSA antibiotic susceptibilities}

Neither CA-MRSA nor HA-MRSA can be treated with $\beta$-lactam antibiotics. However, CA-MRSA tends to be susceptible to more antibiotic classes than HA-MRSA. The former is significantly more susceptible to ciprofloxacin, clindamycin, and gentamicin, and slightly more susceptible to TMP/SMX, than is HA-MRSA [17]. Community-acquired MRSA can be treated with vancomycin, clindamycin, or TMP/SMX; additional agents are tetracycline, linezolid, and gentamicin [12]. Gentamicin should be used only in combination with other agents [12], and some apparently clindamycin-susceptible CA-MRSA strains develop resistance to the drug during therapy [1]. Tigecycline may also be an option for hospitalized patients with CA-MRSA skin infection [12]. 
In patients with presumed CA-MRSA SSTIs, it has been recommended that uncomplicated infections in otherwise healthy individuals be treated empirically with clindamycin, TMP/SMX, or a tetracycline, although specific data from multi-center randomized trials supporting the efficacy of these treatments are lacking [18]. A recent single-center randomized clinical trial compared TMP/SMX and doxycycline for outpatient SSTIs in 34 patients requiring incision and drainage and packing of abscesses, but not hospitalization [19]. This study found an overall clinical failure rate of $9 \%$, with all failures occurring in the TMP/SMX group [19]. In healthy patients with small purulent lesions, drainage alone may be sufficient [1]. However, in patients with cSSTIs and co-morbidities who require hospitalization, initial broadspectrum systemic antibiotic therapy should include specific anti-MRSA activity.

Patients with HA-MRSA infection have a narrower range of therapeutic options. The organism remains sensitive to vancomycin, TMP/SMX, some tetracyclines, and linezolid. Tigecycline and daptomycin also are options. Gentamicin resistance is more common in HA-MRSA than CA-MRSA strains, and most strains are resistant to clindamycin [12].

\section{Antibiotics that stimulate or inhibit toxin production}

Protein cytotoxins play an important role in the pathogenesis of a variety of staphylococcal infections, and toxin production should be considered when selecting an antimicrobial agent for gram-positive pathogens [20]. As many as $54 \%$ of CA-MRSA isolates have the Panton-Valentine leukocidin (PVL) gene, encoding a virulence factor that causes tissue necrosis and leukocyte destruction [21]. Whether PVL contributes to the pathogenesis of necrotizing SSTIs caused by MRSA, or is just a marker, remains to be determined, although results obtained with CA-MRSA isogenic PVL-deletion strains indicate that PVL does not play a major role in CA-MRSA SSTIs [22]. A recently identified class of secreted staphylococcal peptides (phenol-soluble modulin peptides) has a remarkable ability to recruit, activate, and lyse human neutrophils, thus eliminating the main cellular defense against MRSA [23]. The $\beta$-lactam agents actually enhance toxin production and may contribute to worse outcomes in patients with MRSA infections [20]. Linezolid, in contrast, has the ability to inhibit production of PVL and toxic shock syndrome toxin [20].

\section{Vancomycin and Newer Agents for MRSA Infection}

There are four antibiotics approved by the U.S. Food and Drug Administration (FDA) for the treatment of MRSA cSSTIs: Vancomycin, linezolid, daptomycin, and tigecycline [16]. Ceftobiprole and ceftaroline are anti-MRSA cephalosporins under investigation for the treatment of complicated skin and skin structure infections (cSSSIs). The glycopeptides dalbavancin, telavancin, and oritavancin also are under investigation for treatment of cSSSIs, as is iclaprim, a diaminopyrimidine. Table 3 lists the results of clinical trials of these agents in the treatment of MRSA cSSTIs.

\section{Vancomycin}

Vancomycin, a bactericidal glycopeptide, emerged as an important antibiotic in the 1980s and 1990s with the rise of
MRSA infections [24]. Vancomycin has been the reference standard for treating MRSA infections because of its relative safety, its durability against the development of resistance, and-until recently-the lack of other approved alternatives for the treatment of MRSA [25]. However, vancomycin is being linked increasingly to clinical failures, possibly caused by underdosing, poor tissue penetration, loss of accessory gene-regulator function in the organism, slower bactericidal effect, and escalation of vancomycin minimum inhibitory concentrations (MICs) [25,26]. In a single-center review of 288 patients who required surgical intervention for cSSTIs from 2000-2006 in Houston, TX, vancomycin MICs increased in MRSA isolates. In 2003, 100\% of the MRSA isolates from SSTIs had vancomycin MICs $\leq 0.5 \mathrm{mcg} / \mathrm{mL}$, whereas in 2006, 62\% had vancomycin MICs $\leq 0.5 \mathrm{mcg} / \mathrm{mL}$, with $7 \%$ having an MIC of $1 \mathrm{mcg} / \mathrm{mL}$ and $31 \%$ having an MIC of $2 \mathrm{mcg} / \mathrm{mL}$ [27].

Whereas vancomycin resistance is rare, there have been a number of reports of vancomycin-intermediate and vancomycin-resistant S. aureus dating to 1999 [25]. According to the U.S. Centers for Disease Control and Prevention (CDC), overuse of vancomycin and the accompanying emergence of vancomycin resistance are cause for concern [28].

Vancomycin has a relatively low rate of tissue penetration [29-31], typically between $10 \%$ and $20 \%$, sometimes resulting in drug concentrations too low to be therapeutic [29-31]. Vancomycin also has delayed penetration into skin and soft tissues. Vancomycin concentrations in breast tissues were evaluated in 24 women undergoing reconstructive surgery after mastectomy for breast cancer. Patients were given a single prophylactic dose of vancomycin $(1 \mathrm{~g}$ IV) 1-8 h before surgery, and tissue concentrations were measured by high performance liquid chromatography. Vancomycin was not detectable in the majority of patients at 1-3 h postdose [32].

Vancomycin concentrations in serum, tissue, and sternal bone in patients receiving antimicrobial prophylaxis for coronary artery bypass surgery also were examined. The lowest drug concentrations $(4.0-4.8 \mathrm{mcg} / \mathrm{g}$ ) were found in fat when the mean serum concentration was $55.1 \pm 22.8$ $\mathrm{mcg} / \mathrm{mL}$. At $210 \mathrm{~min}$ after vancomycin dosing, the serum concentration decreased to $16.2 \pm 4.6 \mathrm{mcg} / \mathrm{mL}$, with fat concentrations ranging from $5.4-7.7 \mathrm{mcg} / \mathrm{g}$ and skin concentrations ranging from $15.8-23.5 \mathrm{mcg} / \mathrm{g}$, thus documenting delayed tissue penetration of vancomycin [33].

Vancomycin can trigger synergistic nephrotoxicity when administered concurrently with other nephrotoxic agents, and can cause ototoxicity [34]. Additionally, it must be administered parenterally (except in colitis caused by Clostridium difficile) [34], which requires skilled nursing time for IV catheter care, monitoring, and dosage adjustments.

\section{Linezolid}

After vancomycin, linezolid is the second-most-studied agent for MRSA. Linezolid, first in the class of oxazolidinones, offers broad-spectrum gram-positive activity with $100 \%$ oral bioavailability [35]. Linezolid has been approved for the treatment of vancomycin-resistant Enterococcus faecium infections, cSSSIs and nosocomial pneumonia caused by MSSA and MRSA, and uncomplicated SSSIs and com- 
Table 3. Clinical Trial Results for Treatment of Complicated Skin and Soft Tissue Infections Caused by Methicillin-Resistant Staphylococcus aureus

\begin{tabular}{|c|c|c|c|c|c|c|}
\hline Antibiotic & Comparator & $\begin{array}{l}\text { Experimental } \\
\text { design }\end{array}$ & $\begin{array}{c}\text { Total } \\
\text { patients }\end{array}$ & No. of patients & $\begin{array}{l}\text { Outcome in } \\
\text { MRSA } \\
\text { patients: } \\
\text { agent vs. } \\
\text { comparator }\end{array}$ & $\begin{array}{l}\text { Outcome in all } \\
\text { patients: } \\
\text { agent vs. } \\
\text { comparator }\end{array}$ \\
\hline Linezolid $^{42 a}$ & Vancomycin & Open-label & 1,180 & 285 & $\begin{array}{l}\text { Clinical cure }{ }^{43} \text { : } \\
94.0 \% \text { vs. } 83.6 \% \\
\text { Microbiol. cure: } \\
88.6 \% \text { vs. } 66.9 \%\end{array}$ & $\begin{array}{c}\text { Clinically evaluable: } \\
94.4 \% \text { vs. } 90.4 \%\end{array}$ \\
\hline Daptomycin $55 a$ & Vancomycin & Double-blind & 1,092 & 64 & $75.0 \%$ vs. $69.4 \%$ & $\begin{array}{c}\text { Clinically evaluable: } \\
83.4 \% \text { vs. } 84.2 \%\end{array}$ \\
\hline Tigecycline $^{61 a}$ & Vancomycin & Double-blind & 1,116 & 65 & & $\begin{array}{c}\text { Clinically evaluable: } \\
86.5 \% \text { vs. } 88.6 \%\end{array}$ \\
\hline Dalbavancin $68 \mathrm{~b}$ & Linezolid & Double-blind & 854 & 278 & $91 \%$ vs. $89 \%$ & $88.9 \%$ vs. $91.2 \%$ \\
\hline Telavancin ${ }^{72 b}$ & Vancomycin & Double-blind & 1,867 & 579 & $\begin{array}{l}\text { Clinical cure: } \\
90.6 \% \text { vs. } 86.4 \% \\
\text { Microbiol. cure: } \\
90 \% \text { vs. } 85 \%\end{array}$ & $\begin{array}{l}\text { Clinically evaluable: } \\
88 \% \text { vs. } 87 \%\end{array}$ \\
\hline Ceftobiprole ${ }^{64 b}$ & Vancomycin & Double-blind & 784 & 121 & $91.8 \%$ vs. $90.0 \%$ & $\begin{array}{c}\text { Clinical cure ITT: } \\
77.8 \% \text { vs. } 77.5 \% \\
\text { Clinically evaluable: } \\
93.3 \% \text { vs. } 93.5 \%\end{array}$ \\
\hline Ceftobiprole ${ }^{62 b}$ & $\begin{array}{l}\text { Vancomycin }+ \\
\text { ceftazidime }\end{array}$ & $\begin{array}{l}\text { Double-blind } \\
\text { (included } \\
\text { diabetic foot } \\
\text { infections) }\end{array}$ & 828 & 123 & $89.7 \%$ vs. $86.1 \%$ & $\begin{array}{c}\text { Clinical cure ITT: } \\
81.9 \% \text { vs. } 80.8 \% \\
\text { Clinically evaluable: } \\
90.5 \% \text { vs. } 90.2 \%\end{array}$ \\
\hline Iclaprim $^{80 \mathrm{~b}}$ & Linezolid & Double-blind & 497 & $\begin{array}{l}70 \% \text { of } \\
\text { pathogens were } \\
\text { Staphylococcus } \\
\text { aureus, } 25 \% \text { of } \\
\text { which were } \\
\text { MRSA }\end{array}$ & & $\begin{array}{c}\text { Clinical cure ITT: } \\
85.5 \% \text { vs. } 91.9 \% \\
\text { Clinically evaluable: } \\
93.8 \% \text { vs. } 99.1 \% \\
\text { Microbiol. cure: } \\
93.8 \% \text { vs. } 98.8 \%\end{array}$ \\
\hline Iclaprim $^{80 b}$ & Linezolid & Double-blind & 494 & $\begin{array}{l}\sim 60 \% \text { of } \\
\text { pathogens were } \\
\text { S. aureus, } 50 \% \\
\text { of which were } \\
\text { MRSA }\end{array}$ & $\begin{array}{l}\text { Microbiol. cure } \\
\text { MRSA: } \\
77.0 \% \text { vs. } 80.0 \%\end{array}$ & $\begin{array}{l}\text { Clinical cure ITT: } \\
84.9 \% \text { vs. } 87.2 \% \\
\text { Clinically evaluable: } \\
89.6 \% \text { vs. } 96.4 \% \\
\text { Microbiol. cure: } \\
83.5 \% \text { vs. } 84.7 \% \\
\text { MSSA } \\
\text { 77\% vs. } 80 \% \\
\text { MRSA }\end{array}$ \\
\hline
\end{tabular}

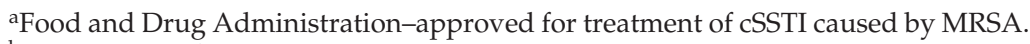

bInvestigational agent.

Microbiol. $=$ microbiological; MRSA $=$ methicillin-resistant Staphylococcus aureus; $I T T=$ intent to treat; MSSA $=$ methicillin-sensitive S. aureus; CSSTI $=$ complicated skin and soft tissue infection.

munity-acquired pneumonia caused by MSSA [35]. Linezolid has demonstrated excellent penetration of bone $(60 \%$, when compared with simultaneous blood concentrations) and muscle (94\%) [36]. In addition, the drug has excellent blister fluid penetration, a mean of $104 \%$, representative of soft tissue penetration [37].

Linezolid soft tissue penetration was examined against strains of MRSA with reduced vancomycin susceptibility in patients with diabetic foot infections [38]. Linezolid concentrations in tissue were found to be $51 \%$ of the simultaneous serum concentrations. Rapid ( $1 \mathrm{~h})$ and prolonged $(12 \mathrm{~h})$ inhibitory activity was observed against each of the study isolates. Furthermore, bactericidal activity was observed for at least $6 \mathrm{~h}(50 \%$ of the dosing interval) against four of the five strains [38]. These findings suggest that linezolid could be effective in the treatment of multi-drug-resistant MRSA, even when the concentration at the infection site is diminished by impaired blood flow.

The 2006 LEADER surveillance program, which assessed more than 5,000 clinical isolates from 50 medical centers throughout the U.S., reported that more than $99.5 \%$ of grampositive organisms and more than $99.9 \%$ of $S$. aureus isolates remain susceptible to linezolid [39]. Recent studies of nosocomial pneumonia and VAP have suggested that linezolid may yield significantly better clinical outcomes than vancomycin in patients with serious infections resulting from MRSA [40,41].

In the largest cSSSI trial to date, 1,200 adult patients with suspected or proved MRSA were randomized to treatment 


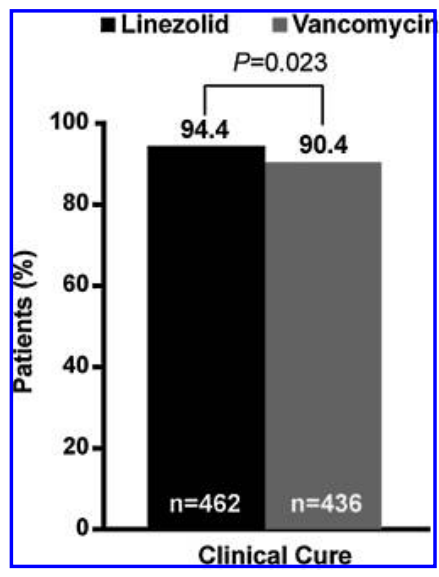

FIG. 2. In the clinically evaluable population of the total study cohort of 1,200 hospitalized adult patients with complicated skin and soft tissue infections, clinical cure was achieved in $94.4 \%(436 / 462)$ of patients treated with linezolid compared with $90.4 \%(394 / 436)$ of patients treated with vancomycin $(\mathrm{p}=0.023)$ [42].

with linezolid (IV or oral) or vancomycin (1 g q12h IV). For the primary efficacy outcome, clinical response at the TOC visit in the ITT population was $92.2 \%(439 / 476)$ for patients treated with linezolid, compared with $88.5 \%$ (402/454) for those treated with vancomycin $(p=0.057)$; thus, the 2 drugs were equivalent in this population (Fig. 2) [42]. For the subgroups with MRSA isolated at baseline in the modified intent-to-treat (mITT) population $(\mathrm{n}=285)$ and in the microbiologically evaluable (ME) population $(\mathrm{n}=361)$, the clinical success (cure) rate was better for linezolid-treated subjects than for the vancomycin-treated subjects (mITT 92.0\% vs. $81.8 \%$; $=0.0114$; ME $94.0 \%$ vs. $83.6 \% ; p=0.0108$ ) (Fig. 3) [43]. The number of subjects with MRSA at baseline was similar in the treatment groups [42]. In this study, microbiologic eradication rates for linezolid $(88.6 \%)$ vs. vancomycin $(66.9 \%$; $\mathrm{p}<0.0001)$ were reported in patients with confirmed MRSA (Fig. 3) [42]. An earlier study in patients with MRSA-complicated surgical site infections also found that significantly more patients treated with linezolid experienced microbiologic success $(87 \%)$ than did patients treated with vancomy$\operatorname{cin}(48 \% ; \mathrm{p}=0.0022)$ [44].

Linezolid, which has a $100 \%$ bioavailable oral formulation, has been associated with shorter LOS and duration of IV treatment compared with vancomycin. A 2005 study found that linezolid-treated patients with cSSSIs caused by suspected or proved MRSA had a hospital LOS 2.5 days shorter than vancomycin-treated patients [45]. A number of other studies have confirmed the cost-effectiveness of linezolid vs. vancomycin for hospitalized patients with cSSTIs [46-50].

In a study of the rates of antimicrobial susceptibility of $S$. aureus from skin and wound infections reported from nine regions of the U.S. during 2005-2007 from The Surveillance Network, linezolid resistance was rare. Linezolid resistance was reported in 13 of 80,527 isolates in 2007, although these data have not been confirmed [51].

Linezolid is not active against gram-negative organisms and appropriate antibiotic coverage should be administered if gram-negative infections are known or suspected [35]. Lactic acidosis has been reported in some patients [35]. Lactic acidosis should be considered in any patient receiving linezolid who presents with nausea, vomiting, and a low serum bicarbonate concentration [35]. Serotonin syndrome also is possible when serotonergic agents, such as selective serotonin reuptake inhibitors (SSRIs), are combined with linezolid [52]. When administering linezolid to patients taking SSRIs, it is prudent to monitor for symptoms such as hyperpyrexia and mental status change [35]. Finally, peripheral and optic neuropathy can occur with linezolid, typically after use for longer than three months [35].

\section{Daptomycin}

Daptomycin, a cyclic lipopeptide, is a potent bactericidal agent that has shown no cross-resistance to date [53,54]. Daptomycin is U.S. Food and Drug Administration (FDA)approved for the treatment of cSSSIs caused by gram-positive pathogens and for bacteremia but not for treatment of pneumonia [54]. Although it is available in IV form only, daptomycin may be administered to outpatients because of its once-daily or every-other-day dosing.

An analysis of 902 evaluable patients from two randomized, multi-national trials demonstrated clinical equivalency between daptomycin and conventional antibiotics (vancomycin or penicillinase-resistant penicillins) in the treatment of cSSSIs [55]. Clinical success rates in the clinically evaluable population were $83.4 \%$ for daptomycin-treated patients and $84.2 \%$ for comparator-treated patients $(95 \% \mathrm{CI}-4.0,5.6)$ [55].

Only 64 patients with MRSA were evaluated microbiologically in the study cohort. Among patients with confirmed MRSA infections, the clinical success rates were $75.0 \%$ for daptomycin and $69.4 \%$ for the comparator drug (95\% CI $-28.5,17.4$ ) [55]. The frequency, distribution, and severity of adverse events (AEs) were similar for in the two treatment groups [55].

The efficacy of daptomycin in cSSTIs also has been examined in the Cubicin Outcomes Registry and Experience 2004 Registry, a multi-center observational registry involving 45 institutions. A total of 165 patients were identified, including 145 with MRSA and 20 with MSSA cSSTIs, but with-

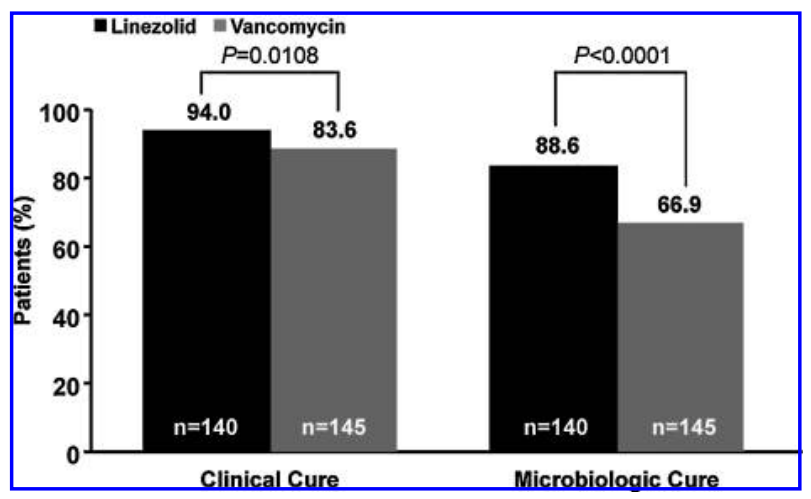

FIG. 3. In microbiologically evaluable patients with methicillin-resistant Staphylococcus aureus isolated as causative pathogen for complicated skin and soft tissue infections, linezolid treatment was associated with higher clinical $(94.0 \%$ vs. $83.6 \%$; $p=0.0108)$ and microbiologic $(88.6 \%$ vs. $66.9 \%$; $\mathrm{p}<0.0001)$ cure rates $[42,43]$. 
out bacteremia, endocarditis, osteomyelitis, or other major infectious processes. Clinical success was achieved with daptomycin in $89.1 \%$ of patients overall, including $89.7 \%$ in patients with MRSA. Prior antibiotic therapy had been administered to $74.2 \%$ of patients and concomitant antibiotic therapy to $39.4 \%$ [56].

Another study examined daptomycin efficacy in 53 adult patients with cSSTIs at risk for MRSA infection compared with a matched retrospective cohort of 212 patients treated with vancomycin. The proportions of patients with clinical improvement or resolution of their infections on days three and five were $90 \%$ vs. $70 \%$ and $98 \%$ vs. $81 \%$ in the daptomycin and vancomycin groups, respectively [57].

The serum creatine phosphokinase concentration should be monitored weekly during use of daptomycin, especially if high doses are given [54]. Caution is necessary in patients previously treated with vancomycin, which may influence daptomycin susceptibility [58].

\section{Tigecycline}

Tigecycline, approved in 2005, is the first agent of the glycylcycline class. Chemically similar to minocycline, tigecycline is better tolerated and more active against tetracycline-resistant strains [59]. Tigecycline is effective over a broader spectrum than many other agents, but does not cover Pseudomonas aeruginosa or Proteus spp. [60]. In a study of more than 500 gram-positive isolates, tigecycline inhibited all strains, including those resistant to other tetracyclines [59]. Its coverage includes VRE, penicillin-resistant S. pneumoniae, and MRSA [59].

In two phase 3, double-blind studies of hospitalized patients with cSSSIs, tigecycline demonstrated clinical cure rates equivalent to those of vancomycin plus aztreonam among the 833 clinically evaluable patients ( $86.5 \%$ vs. $88.6 \%$, respectively; $95 \% \mathrm{CI}-6.8,2.7)$ [61]. In these two registration studies, only 65 patients with MRSA were ME [61]. Among the ME patients with MRSA infection, the microbiologic eradication rates were $78.1 \%$ for tigecycline-treated patients and $75.8 \%$ for vancomycin-treated patients [61]. Each medication regimen was well tolerated. More AEs related to the digestive tract were reported in the tigecycline group, and more rash, cardiovascular events, and increases in hepatic aminotransferase concentrations were reported in the vancomycin/aztreonam group [61]. In clinical trials, the most frequent side effects associated with tigecycline were nausea and vomiting [60].

\section{Investigational cephalosporins}

The cephalosporin ceftobiprole, which is not yet approved by the FDA, is the first $\beta$-lactam antibiotic to have activity against MRSA, as well as penicillin-resistant streptococci. Ceftobiprole has shown low potential to select for resistance [25]. The drug also appears to have clinically relevant activity against gram-negative bacteria [62]. In testing against 100 CA-MRSA and 100 HA-MRSA isolates, the documented $\mathrm{MIC}_{50} / \mathrm{MIC}_{90}$ values were $0.5 \mathrm{mcg} / \mathrm{mL}$ against both types. In time-kill analysis, ceftobiprole was bactericidal at all concentrations tested [63].

In a randomized, multi-center, global, double-blind trial comparing ceftobiprole with vancomycin plus ceftazidime in 729 clinically evaluable patients with cSSSIs (including dia- betic foot infections), the clinical cure rate at the TOC was $90.5 \%$ for ceftobiprole-treated and $90.2 \%$ for comparatortreated patients $(95 \% \mathrm{CI}-4.2,4.9)$ [62]. In patients with MRSA infection, the clinical cure rate was $89.7 \%$ for ceftobiprole-treated and $86.1 \%$ for comparator-treated patients $(95 \% \mathrm{CI}-8.0,19.7)$ [62]. Ceftobiprole was well-tolerated, and the incidence of serious AEs was similar in the two treatment groups [62].

A second global, randomized, double-blind trial compared the efficacy of ceftobiprole with that of vancomycin in patients with cSSTIs caused by gram-positive bacteria [64]. The primary objective was to assess non-inferiority on the basis of the cure rate seven to 14 days after the completion of therapy in patients receiving ceftobiprole $500 \mathrm{mg}$ q12h or vancomycin $1 \mathrm{~g} \mathrm{q12h}$. Of 784 patients randomized, 282 who received ceftobiprole and 277 who received vancomycin were evaluable clinically. Of these patients, $93.3 \%$ treated with ceftobiprole and $93.5 \%$ treated with vancomycin were cured $(95 \%$ CI $-4.4,3.9)$. The cure rates for patients with MRSA infections were $91.8 \%(56 / 61)$ with ceftobiprole and $90.0 \%(54 / 60)$ with vancomycin $(95 \%$ CI $-8.4,12.1)$ [64]. At least one AE was reported by $52 \%$ of the ceftobiprole-treated patients and $51 \%$ of the vancomycin-treated patients. The most common AEs reported by the ceftobiprole-treated patients were nausea $(14 \%)$ and taste disturbance $(8 \%)$. Discontinuation of the study drug because of treatmentassociated AEs occurred in $4 \%(n=17)$ of the ceftobiproletreated patients and $6 \%(\mathrm{n}=22)$ of the vancomycin-treated patients [64].

The results of these two trials support the use of ceftobiprole as a treatment option for patients with cSSSIs caused by a spectrum of gram-positive bacteria. Ceftobiprole has received fast-track designation from the FDA for the treatment of cSSSIs caused by MRSA, with an additional designation for the treatment of hospital-acquired pneumonia and VAP caused by proved or suspected MRSA [65]. In March, 2008, the FDA issued a letter requesting additional information for ceftobiprole in the treatment of cSSTIs, including diabetic foot infections. According to Basilea Pharmaceutica, FDA approval is subject to clinical study site inspections, the assessment of clinical and microbiological data, "and further characterization of patients with diabetic foot infections" [66].

Ceftaroline, another investigational anti-MRSA cephalosporin with broad-spectrum coverage for gram-negative and gram-positive pathogens, is under investigation for cSSSIs [25]. In a phase 2 study, the clinical cure rate in the clinically evaluable population was $96.7 \%$ for ceftaroline-treated patients and $88.9 \%$ for patients receiving vancomycin with or without aztreonam [67]. Among the ME subjects, the microbiological success rate was $95.2 \%$ for ceftaroline vs. $85.7 \%$ for vancomycin. Ceftaroline exhibited a favorable safety and tolerability profile, consistent with that of marketed cephalosporins [67].

\section{Investigational glycopeptides}

There are three anti-MRSA IV glycopeptides in late development: Dalbavancin, telavancin, and oritavancin.

Dalbavancin. The unique feature of dalbavancin is its extraordinarily long half-life (6-10 days), which allows once- 
weekly dosing [25]. In a phase 3 trial, IV dalbavancin, administered on day 1 and day 8, was compared with IV/oral linezolid, given twice daily for 14 days, in 660 clinically evaluable patients with cSSSIs [68]. At the TOC visit, $88.9 \%$ of the dalbavancin-treated patients and $91.2 \%$ of the linezolid-treated patients had achieved clinical success [68]. The rates of MRSA eradication in 278 patients with confirmed MRSA cSSTIs were $91 \%$ in the dalbavancin group and $89 \%$ in the linezolid group [68]. The safety profiles for the two agents were similar [68].

Telavancin. Telavancin is a semisynthetic lipoglycopeptide with a dual mechanism of action: Inhibition of cell wall synthesis and disruption of membrane barrier function. It has a 7- to 9-h half-life, which allows once-daily dosing. Telavancin is under investigation for the treatment of cSSTIs, nosocomial pneumonia, and uncomplicated bacteremia caused by S. aureus [25]. In two phase 2 trials for treatment of cSSTIs, similar clinical success rates were achieved in patients receiving telavancin or standard therapy for infections caused by $S$. aureus and MRSA $[69,70]$.

Two parallel, randomized, double-blind, active-control, phase 3 studies with a pre-specified pooled analysis design were conducted in patients aged 18 years or older who had cSSTIs caused by suspected or confirmed gram-positive organisms [71]. Patients were randomized to receive either telavancin (10 mg/kg IV q24h) or vancomycin (1 g IV q12h). A total of 1,867 patients were randomized and received at least one dose of study medication. In the clinically evaluable population, at 7-14 days after receipt of the last antibiotic dose, success was achieved in $88 \%$ and $87 \%$ of patients who received telavancin and vancomycin, respectively $(95 \%$ CI -2.1, 4.6) [71].

Methicillin-resistant $S$. aureus was isolated at baseline from samples from 579 clinically evaluable patients-the largest series to date. Among these patients, the cure rate was $91 \%$ among patients who received telavancin and 86\% among patients who received vancomycin $(95 \% \mathrm{CI}-1.1,9.3)$. Microbiologic eradication of MRSA was achieved in $90 \%$ of the telavancin group and $85 \%$ of the vancomycin group ( $95 \% \mathrm{CI}-0.9$, 9.8). This study confirmed that telavancin given once daily is at least as effective as vancomycin for the treatment of patients with cSSTIs, including those infected with MRSA [71].

Therapy was discontinued because of AEs in $8 \%$ and $6 \%$ of patients who received telavancin and vancomycin, respectively. Except for mild taste disturbance, nausea, vomiting, and serum creatinine concentration elevation in the telavancin treatment group and pruritus in the vancomycin treatment group, the AEs were similar in the two groups with regard to type and severity [71].

Oritavancin. Oritavancin is another broad-spectrum semisynthetic glycopeptide under development for the treatment of cSSTIs, catheter-related blood stream infections, and nosocomial pneumonia [25]. It has demonstrated activity against vancomycin-resistant strains of staphylococci and enterococci [25] and has a long half-life (100 h), which is expected to allow once-daily or every-other-day dosing. The manufacturer reports that two phase 3 trials for treatment of cSSTIs have been completed, with the primary endpoints being met in each $[72,73,74]$.

\section{Iclaprim}

Iclaprim, an agent under investigation, is a diaminopyrimidine with activity against MRSA [25]. The manufacturer reports that in two phase 3 trials for the treatment of cSSTIs, iclaprim achieved its primary efficacy endpoint and had a safety profile similar to that of the comparator, linezolid [75]. The multi-center, double-blind, randomized, active-control, parallel-assignment, phase 3 trial, ASSIST-1 (Arpida's Skin and Skin Structure Infection Study), compared IV iclaprim $(0.8 \mathrm{mg} / \mathrm{kg} ; \mathrm{N}=250)$ with IV linezolid (600 mg; $\mathrm{N}=247)$, both administered for 10 to 14 days, in patients with cSSTI who had extensive cellulitis, abscesses, ulcers, burns, or wounds [76,77]. The primary objective was to compare the clinical cure rates of iclaprim and linezolid at the TOC visit [77]. Secondary outcomes included clinical efficacy at the end of the trial and clinical outcomes of the ME and ITT populations [77]. Approximately $70 \%$ of the pathogens isolated at baseline were S. aureus, 25\% of which were MRSA [77]. For the ME patients, the cure rates were $94.7 \%$ and $98.8 \%$ for iclaprim and linezolid, respectively. Non-inferiority was achieved for the primary endpoint, and the overall clinical cure rate for the ITT population at the TOC was $85.5 \%$ and $91.9 \%$ for iclaprim and linezolid, respectively. The clinically evaluable patients had cure rates of $93.8 \%$ and $99.1 \%$, respectively [77].

A subsequent multi-center, double-blind, randomized, active-control, parallel-assignment phase 3 clinical trial, ASSIST-2, was initiated in cSSTI patients with extensive cellulitis, abscesses, ulcers, burns, or wounds to compare IV iclaprim $(n=251)$ with IV linezolid $(n=243)$ [77,78]. The primary and secondary endpoints were the same as those in the ASSIST-1 trial. Preliminary analysis indicated overall clinical cure rates for the ITT population of $84.9 \%$ and $87.2 \%$ for iclaprim and linezolid, respectively [77]. The most common baseline pathogen was S. aureus $(\approx 60 \%), 50 \%$ of which were MRSA. The microbiological eradication rates for MSSA were $83.5 \%$ and $84.7 \%$ for iclaprim and linezolid, respectively, and $77.0 \%$ and $80.0 \%$ for MRSA, respectively [77]. For patients with gram-positive pathogen infections at baseline, the clinical cure rates were $83.3 \%$ and $85.9 \%$ for iclaprim and linezolid, respectively. In a preliminary analysis of the clinically evaluable population, the cure rates were $89.6 \%$ and $96.4 \%$ for iclaprim and linezolid, respectively [77]. Further analysis of the trial results is ongoing $[77,78]$.

\section{Conclusions}

Inadequate treatment of severe infections in hospitalized patients contributes to in-hospital death and prolonged LOS. Prompt, appropriate treatment of cSSTIs caused by MRSA increases the chances of a successful outcome. The choice of antimicrobial agent for empiric treatment of cSSTI should be guided by a number of considerations, including the site and type of infection, presence of immunocompromised state or neutropenia, and risk factors for HA-MRSA or CA-MRSA infection. Patients with severe infection or co-morbidities should be treated aggressively with empiric broad-spectrum antimicrobial therapy and then de-escalated to narrowerspectrum agents depending on the culture findings and clinical response. It is of paramount importance to obtain specimens for culture and antimicrobial susceptibilities 
given the high prevalence of MRSA as a causative pathogen in cSSTIs.

Community-associated MRSA infections are susceptible to more antibiotics than HA-MRSA infections, which commonly are multi-drug-resistant. Most CA-MRSA strains remain susceptible to ciprofloxacin, clindamycin, gentamicin, and TMP/SMX, although resistance to clindamycin can emerge during treatment. Hospital-acquired MRSA is susceptible to fewer antibiotics. Of the agents available currently, vancomycin has been the reference standard, but increasing clinical failures have been reported-likely as a result of poor tissue penetration and increasing MICs. Alternative agents for the treatment of HA-MRSA include linezolid, daptomycin, and tigecycline, which have been well-studied and are approved by the FDA for the treatment of cSSTIs. A number of other antibiotic agents are under development for the treatment of cSSTIs caused by MRSA.

\section{References}

1. Gorwitz RJ, Jernigan DB, Powers JH, Jernigan JA; Participants in the CDC-Convened Experts' Meeting on Management of MRSA in the Community. Strategies for clinical management of MRSA in the community: Summary of an experts' meeting convened by the Centers for Disease Control and Prevention. 2006. Available at http://198.246.98.21/ ncidod/dhqp/pdf/ar/CAMRSA_ExpMtgStrategies.pdf. Accessed March 10, 2008. (//www.cdc.gov/ncidod/dhqp/ ar_mrsa_ca_04meeting.html.)

2. Kuo DC, Chasm RM, Witting MD. Emergency department physician ability to predict methicillin-resistant Staphylococcus aureus skin and soft tissue infections. J Emerg Med 2008 May 29 [Epub ahead of print].

3. Kollef MH, Sherman G, Ward S, Fraser VJ. Inadequate antimicrobial treatment of infections: A risk factor for hospital mortality among critically ill patients. Chest 1999;115: 462-474.

4. Ruhe JJ, Smith N, Bradsher RW, Menon A. Community-onset methicillin-resistant Staphylococcus aureus skin and softtissue infections: Impact of antimicrobial therapy on outcome. Clin Infect Dis 2007;44:777-784.

5. Iregui M, Ward S, Sherman G, et al. Clinical importance of delays in the initiation of appropriate antibiotic treatment for ventilator-associated pneumonia. Chest 2002;122:262-268.

6. Garnacho-Montero J. Impact of adequate empirical antibiotic therapy on the outcome of patients admitted to the intensive care unit with sepsis. Crit Care Med 2003;31: 2742-2751.

7. Alvarez-Lerma F; ICU-Acquired Pneumonia Study Group. Modification of empiric antibiotic treatment in patients with pneumonia acquired in the intensive care unit. Intensive Care Med 1996;22:387-394.

8. Lodise TP, McKinnon PS, Swiderski L, Rybak MJ. Outcomes analysis of delayed antibiotic treatment for hospital-acquired Staphylococcus aureus bacteremia. Clin Infect Dis 2003;36:1418-1423.

9. Schramm GE, Johnson JA, Doherty JA, et al. Methicillin-resistant Staphylococcus aureus sterile-site infection: The importance of appropriate initial antimicrobial treatment. Crit Care Med 2006;34:2069-2074.

10. Chuck EA, Frazee BW, Lambert L, McCabe R. The benefit of empiric treatment for methicillin-resistant Staphylococcus aureus. J Emerg Med 2008 May 29 [Epub ahead of print]
11. Miller LG, Quan C, Shay A, et al. A prospective investigation of outcomes after hospital discharge for endemic, communityacquired methicillin-resistant and -susceptible Staphylococcus aureus skin infection. Clin Infect Dis 2007;44:483-492.

12. Merlino JI, Malangoni MA. Complicated skin and soft-tissue infections: Diagnostic approach and empiric treatment options. Cleve Clin J Med 2007;74(Suppl 4):S21-S28.

13. Stevens DL, Bisno AL, Chambers HF, et al; Infectious Diseases Society of America. Practice guidelines for the diagnosis and management of skin and soft-tissue infections. Clin Infect Dis 2005;41:1373-1406.

14. Adeyemi OA, Qi C, Zembower TR, et al. Invasive infections with community-associated methicillin-resistant Staphylococcus aureus after kidney transplantation. I Clin Microbiol 2008;46:2809-2813.

15. Fridkin SK, Hageman JC, Morrison M, et al; Active Bacterial Core Surveillance Program of the Emerging Infections Program Network. Methicillin-resistant Staphylococcus aureus disease in three communities. N Engl J Med 2005;352: 1436-1444.

16. Daum RS. Skin and soft-tissue infections caused by methicillin resistant Staphylococcus aureus. N Engl J Med 2007; 357:380-390.

17. Naimi TS, LeDell KH, Como-Sabetti K, et al. Comparison of community- and health care-associated methicillin-resistant Staphylococcus aureus infection. JAMA 2003;290:2976-2984.

18. Stryjewski ME, Chambers HF. Skin and soft-tissue infections caused by community-acquired methicillin-resistant Staphylococcus aureus. Clin Infect Dis 2008;46(Suppl): S368-S377.

19. Cenizal MJ, Skiest D, Luber S, et al. Prospective randomized trial of empiric therapy with trimethoprim-sulfamethoxazole or doxycycline for outpatient skin and soft tissue infections in an area of high prevalence of methicillin-resistant Staphylococcus aureus. Antimicrob Agents Chemother 2007; 51:2628-2630.

20. Stevens DL, Ma Y, Salmi DB, et al. Impact of antibiotics on expression of virulence-associated exotoxin genes in methicillin-sensitive and methicillin-resistant Staphylococcus aureus. J Infect Dis 2007;195:202-211.

21. Davis SL, Perri MB, Donabedian SM, et al. Epidemiology and outcomes of community-associated methicillin-resistant Staphylococcus aureus infection. I Clin Microbiol 2007;45: 1705-1711.

22. Voyich JM, Otto M, Mathema B, et al. Is Panton-Valentine leukocidin the major virulence determinant in communityassociated methicillin-resistant Staphylococcus aureus disease? J Infect Dis 2006;194:1761-1770.

23. Wang R, Braughton KR, Kretschmer D, et al. Identification of novel cytolytic peptides as key virulence determinants for community-associated MRSA. Nat Med 2007;13: 1418-1420.

24. Kirst HA, Thompson DG, Nicas TI. Historical yearly usage of vancomycin. Antimicrob Agents Chemother 1998;42: 1303-1304.

25. Micek ST. Alternatives to vancomycin for the treatment of methicillin-resistant Staphylococcus aureus infections. Clin Infect Dis 2007;45:S184-190.

26. Stevens DL. The role of vancomycin in the treatment paradigm. Clin Infect Dis 2006;42(suppl 1):S51-S57.

27. Awad SS, Elhabash SI, Lee L, et al. Increasing incidence of methicillin-resistant Staphylococcus aureus skin and soft-tissue infections: Reconsideration of empiric antimicrobial therapy. Am J Surg 2007;194:606-610. 
28. Centers for Disease Control and Prevention. 12 Steps to Prevent Antimicrobial Resistance among Hospitalized Adults. CDC Campaign to Prevent Antimicrobial Resistance in Healthcare Settings. Available at http://www.cdc.gov/drug resistance/healthcare/ha/12steps_HA.htm. Accessed August 2, 2006.

29. Daschner FD, Frank U, Kümmel A, et al. Pharmacokinetcs of vancomycin in serum and tissue of patients undergoing open-heart surgery. J Antimicrob Chemother 1987;19: 359-362.

30. Cruciani M, Gatti G, Lazzarini L, et al. Penetration of vancomycin into human lung tissue. I Antimicrob Chemother 1996;38:865-869.

31. Graziani AL, Lawson LA, Gibson GA, et al. Vancomycin concentrations in infected and noninfected human bone. Antimicrob Agents Chemother 1988;32:1320-1322.

32. Luzzati R, Sanna A, Allegranzi B, et al. Pharmacokinetics and tissue penetration of vancomycin in patients undergoing prosthetic mammary surgery. I Antimicrob Chemother 2000;45:243-245.

33. Kitzes-Cohen R, Farin D, Piva G, et al. Pharmacokinetics of vancomycin administered as prophylaxis before cardiac surgery. Therapeutic Drug Monit 2000;22:661-667.

34. Vancocin [package insert]. Exton, PA. ViroPharma Inc. 2005.

35. Zyvox [package insert]. New York, NY. Pfizer Inc. 2007.

36. Lovering AM, Zhang J, Bannister GC, et al. Penetration of linezolid into bone, fat, muscle and haematoma of patients undergoing routine hip replacement. I Antimicrob Chemother 2002;50:73-77.

37. Gee T, Ellis R, Marshall G, et al. Pharmacokinetics and tissue penetration of linezolid following multiple oral doses. Antimicrob Agents Chemother 2001;45:1843-1846.

38. Stein GE, Schooley S, Peloquin CA, et al. Linezolid tissue penetration and serum activity against strains of methicillinresistant Staphylococcus aureus with reduced vancomycin susceptibility in diabetic patients with foot infections. I Antimicrob Chemother 2007;60:819-823.

39. Jones RN, Fritsche TR, Sader HS, Ross JE. LEADER surveillance program results for 2006: An activity and spectrum analysis of linezolid using clinical isolates from the United States (50 medical centers). Diagn Microbiol Infect Dis 2007;59:309-317.

40. Wunderink RG, Rello J, Cammarata SK, et al. Linezolid vs vancomycin: Analysis of two double-blind studies of patients with methicillin-resistant Staphylococcus aureus nosocomial pneumonia. Chest 2003;124:1789-1797.

41. Kollef MH, Rello J, Cammarata SK, et al. Clinical cure and survival in gram-positive ventilator-associated pneumonia: Retrospective analysis of two double-blind studies comparing linezolid with vancomycin. Intensive Care Med 2004;30:388-394.

42. Weigelt J, Itani K, Stevens D, et al; Linezolid CSSTI Study Group. Linezolid versus vancomycin in treatment of complicated skin and soft tissue infections. Antimicrob Agents Chemother 2005;49:2260-2266.

43. Weigelt J, Itani K, Stevens D, Knirsch C. Is linezolid superior to vancomycin for complicated skin and soft tissue infections due to methicillin-resistant Staphylococcus aureus? Antimicrob Agents Chemother 2006;50:1910-1911.

44. Weigelt J, Kaafarani HM, Itani KM, Swanson RN. Linezolid eradicates MRSA better than vancomycin from surgical-site infections. Am J Surg 2004;188:760-766.

45. Itani KMF, Weigelt J, Li JZ, Duttagupta S. Linezolid reduces length of stay and duration of intravenous treatment com- pared with vancomycin for complicated skin and soft tissue infections due to suspected or proven methicillin-resistant Staphylococcus aureus (MRSA). Int J Antimicrob Agents 2005;26:442-448.

46. Schürmann D, Sorensen SV, De Cock E, et al. Cost-effectiveness of linezolid versus vancomycin for hospitalised patients with complicated skin and soft-tissue infections in Germany. Eur J Health Econ 2008 Apr 24 [Epub ahead of print].

47. McCollum M, Sorensen SV, Liu LZ. A comparison of costs and hospital length of stay associated with intravenous/oral linezolid or intravenous vancomycin treatment of complicated skin and soft-tissue infections caused by suspected or confirmed methicillin-resistant Staphylococcus aureus in elderly US patients. Clin Ther 2007;29:469-477.

48. McKinnon PS, Sorensen SV, Liu LZ, Itani KMF. Impact of linezolid on economic outcomes and determinants of cost in a clinical trial evaluating patients with MRSA complicated skin and soft-tissue infections. Ann Pharmacother 2006;40: 1017-1023.

49. Sharpe JN, Shively, Polk HC Jr. Clinical and economic outcomes of oral linezolid versus intravenous vancomycin in the treatment of MRSA-complicated, lower-extremity skin and soft-tissue infections caused by methicillin-resistant Staphylococcus aureus (MRSA). Am J Surg 2005;189:425-428.

50. Li JZ, Willke RJ, Rittenhouse BE, Rybak MJ. Effect of linezolid versus vancomycin on length of hospital stay in patients with complicated skin and soft tissue infections caused by known or suspected methicillin-resistant staphylococci: Results from a randomized clinical trial. Surg Infect 2003;4:57-70.

51. Tillotson GS, Draghi DC, Sahm DF, et al. Susceptibility of Staphylococcus aureus isolated from skin and wound infections in the United States 2005-07: Laboratory-based surveillance study. I Antimicrob Chemother 2008;62:109-115.

52. Narita $\mathrm{M}$ et al. Linezolid-associated peripheral and optic neuropathy, lactic acidosis, and serotonin syndrome. Pharmacotherapy 2007;27:1189-1197.

53. Owens RC Jr, Lamp KC, Friedrich LV, Russo R. Postmarketing clinical experience in patients with skin and skinstructure infections treated with daptomycin. Am J Med 2007;120(10 suppl 1):S6-S12.

54. Cubicin [package insert]. Lexington, MA. Cubist Pharmaceuticals, Inc. 2007.

55. Arbeit RD, Maki D, Tally FP, et al.; Daptomycin 98-01 and 99-01 Investigators. The safety and efficacy of daptomycin for the treatment of complicated skin and skin-structure infections. Clin Infect Dis 2004;38:1673-1681.

56. Martone WJ, Lamp KC. Efficacy of daptomycin in skin and skin-structure infections due to methicillin-sensitive and -resistant Staphylococcus aureus: Results from the CORE Registry. Curr Med Res Opin 2006;22:2337-2343.

57. Davis SL, McKinnon PS, Hall LM, et al. Daptomycin versus vancomycin for complicated skin and skin structure infections: Clinical and economic outcomes. Pharmacotherapy 2007;27:1611-1618.

58. Sakoulas G, Alder J, Thauvin-Eliopoulos C, et al. Induction of daptomycin heterogeneous susceptibility in Staphylococcus aureus by exposure to vancomycin. Antimicrob Agents Chemother 2006;50:1581-1585.

59. Boucher HW, Wennersten CB, Eliopoulos GM. In vitro activities of the glycylcycline GAR-936 against gram-positive bacteria. Antimicrob Agents Chemother 2000;44:2225-2229.

60. Tygacil [package insert]. Philadelphia, PA. Wyeth Pharmaceuticals Inc. 2007. 
61. Ellis-Grosse EJ, Babinchak T, Dartois N, et al.; Tigecycline 300 and 305 cSSSI Study Groups. The efficacy and safety of tigecycline in the treatment of skin and skin-structure infections: Results of 2 double-blind phase 3 comparison studies with vancomycin-aztreonam. Clin Infect Dis 2005; 41(suppl 5):S341-S353.

62. Noel GJ, Bush K, Bagchi P, et al. A randomized, doubleblind trial comparing ceftobiprole medocaril with vancomycin plus ceftazidime for the treatment of patients with complicated skin and skin-structure infections. Clin Infect Dis 2008;46:647-655.

63. Leonard SN, Cheung CM, Rybak MJ. Activities of ceftobiprole, linezolid, vancomycin, and daptomycin against community-associated and hospital-associated methicillin-resistant Staphylococcus aureus. Antimicrob Agents Chemother 2008;52:2974-2976.

64. Noel GJ, Strauss RS, Amsler K, et al. Results of a doubleblind, randomized trial of ceftobiprole treatment of complicated skin and skin structure infections caused by gram-positive bacteria. Antimicrob Agents Chemother 2008;52:37-44.

65. Ceftobiprole (BAL5788) [Web site]. Basel, Switzerland: Basilea Pharmaceutica Ltd; 2008. Available at http:// www.basilea.com/template_loader.php?tplpage_id=19\&_f unction $=$ render\&id $=1$. Accessed January 3, 2008.

66. FDA issues approvable letter for ceftobiprole, a new anti-MRSA broad-spectrum antibiotic [press release]. Basel, Switzerland: Basilea Pharmaceutica Ltd; March 18, 2008. Available at http://www.basilea.com/template_loader.php?tplpage_ id $=34 \&$ mode $=$ details\&id $=172$. Accessed June 17, 2008.

67. Talbot GH, Thye D, Das A, Ge Y. Phase 2 study of ceftaroline versus standard therapy in treatment of complicated skin and skin structure infections. Antimicrob Agents Chemother 2007;51:3612-3616.

68. Jauregui LE, Babazadeh S, Seltzer E, et al. Randomized, double-blind comparison of once-weekly dalbavancin versus twice-daily linezolid therapy for the treatment of complicated skin and skin structure infections. Clin Infect Dis 2005;41:1407-1415.

69. Stryjewski ME, O'Riordan WD, Lau WK, et al.; FAST Investigator Group. Telavancin versus standard therapy for treatment of complicated skin and soft-tissue infections due to gram-positive bacteria. Clin Infect Dis 2005;40:1601-1607.

70. Stryjewski ME, Chu VH, O'Riordan WD, et al.; FAST 2 Investigator Group. Telavancin versus standard therapy for treatment of complicated skin and skin structure infections caused by gram-positive bacteria: FAST 2 study. Antimicrob Agents Chemother 2006;50:862-867.

71. Stryjewski ME, Graham DR, Wilson SE, et al. on behalf of the Assessment of Telavancin in Complicated Skin and SkinStructure Infections Study. Telavancin versus vancomycin for the treatment of complicated skin and skin-structure infections caused by gram-positive organisms. Clin Infect Dis 2008;46:1683-1693.

72. Targanta Therapeutics. Pipeline: Oritavancin program [Targanta.com Web site]. Available at http://www.targanta. com/pipeline/oritavancin.html. Accessed June 18, 2008.

73. Giamarellou H, O'Riordan W, Harris H, Owen S, Porter S, Loutit J. Phase 3 trial comparing 3-7 days of oritavancin vs. 10-14 days of vancomycin/cephalexin in the treatment of patients with complicated skin and skin structure infections (cSSSI) [abstract]. Presented at: 43rd Interscience Conference on Antimicrobial Agents and Chemotherapy (ICAAC); September 14-17, 2003; Chicago, IL. Abstract L-739a.

74. Wasilewski MM, Disch DP, McGill JM, Harris HW, O'Riordan W, Zeckel ML. Equivalence of shorter course therapy with oritavancin vs. vancomycin/cephalexin in complicated skin/skin structure infections (CSSI) [abstract]. Presented at: 41st Interscience Conference on Antimicrobial Agents and Chemotherapy (ICAAC); December 16-19, 2001; Chicago, IL. Abstract UL-18.

75. Arpida provides comprehensive overview of pivotal phase III trial data [press release]. Reinach, Switzerland: Arpida Ltd.; April 17, 2008. Available at http://www.arpida. ch/users/1/content/arpida-assist-overview.pdf. Accessed April 18, 2008.

76. Arpida's Skin and Skin Structure Infection Study 1 (ASSIST1). Phase 3 safety and efficacy study of IV iclaprim vs linezolid in cSSSI (ASSIST-1). Available at http://clinicaltrials. gov/ct2/show / NCT00299520?term =iclaprim +AND+skin \&rank=1. Accessed June 18, 2008.

77. Peppard WJ, Schuenke CD. Iclaprim, a diaminopyrimidine dihydrofolate reductase inhibitor for the potential treatment of antibiotic-resistant staphylococcal infections. Curr Opin Invest Drugs 2008;9:210-225.

78. Arpida's Skin and Skin Structure Infection Study 2 (ASSIST2). Study of intravenous (IV) iclaprim versus linezolid in complicated skin and skin structure infections [cSSSI] (ASSIST-2). Available at http://clinicaltrials.gov/ct2/show/ NCT00303550. Accessed June 18, 2008.

Address reprint requests to: Dr. Lena M. Napolitano Department of Surgery University of Michigan Health System Room 1C421, University Hospital 1500 East Medical Center Dr. Ann Arbor, MI 48109-0033

E-mail: lenan@umich.edu 